\title{
Uso das Ferramentas APF e COCOMO para Estimativa da Capacidade Produtiva da TI
}

\author{
Angélica Toffano Seidel Calazans \\ Caixa Econômica Federal \\ angélica.calazans@caixa.gov.br
}

\author{
Marcelo Antonio Lopes de Oliveira \\ Politec Ltda. \\ marceloo@bsb.politec.com.br
}

\begin{abstract}
Resumo
Estimar a capacidade produtiva da Tecnologia da Informação (TI) para atender ao Planejamento Estratégico da empresa é uma necessidade constante. A TI necessita identificar a sua capacidade produtiva e estimar a necessidade futura de produção de sistemas para conseguir alinhar seus objetivos com os objetivos estratégicos da empresa. Este documento apresenta um relato de experiência da aplicação de um processo de estimativa da capacidade produtiva da TI, tendo como base o planejamento estratégico de uma instituição financeira do governo federal. Na elaboração destas estimativas foram utilizadas as métricas Análise por Pontos de Função APF e COnstructive COst Model - COCOMO.
\end{abstract}

Palavras chaves - capacidade produtiva, estimativa, APF, COCOMO

\begin{abstract}
Information Technology (IT) productive capacity estimation to attend to the Companies Strategic Planning is a constant need. IT needs to identify the productive capacity and estimate the systems future need to align its objective with the company strategic objectives. This document presents an experience from the IT productive capacity estimation trial application, using a government financial institution strategic planning. The Function Points Analysis - FPA and COnstructive COst Model - COCOMO metrics were utilized to elaborate these estimations.
\end{abstract}

Key Words - Productive Capacity, estimative, FPA, COCOMO

\section{Introdução}

Um dos problemas da TI é identificar a sua capacidade produtiva e estimar a necessidade futura de produção de sistemas de forma a conseguir alinhar seus objetivos com os objetivos estratégicos da empresa.

Taurion [9] ressalta que o sucesso do uso de TI depende do grau de alinhamento ${ }^{1}$ entre objetivos da área de TI e objetivos da área de negócio.

Considerando a necessidade da TI de identificar sua capacidade produtiva, presente e futura, para desta forma melhor entender, controlar e aperfeiçoar o processo de produção de software e conseqüentemente alinhar os seus objetivos aos objetivos estratégicos da empresa, no escopo deste trabalho será apresentada a aplicação da APF e do COCOMO para levantamento da estimativa da capacidade produtiva da TI em uma instituição financeira do governo federal.

O documento está organizado nas seguintes seções: breves conceitos de medição e dos métodos APF e COCOMO (seção 2), aplicação da proposta (seção 3), conclusões e sugestões de melhoria do processo (seção 4).

${ }^{1}$ De acordo com Boar [1], alinhamento é a coordenação, colaboração e perseverança em direção a um conjunto compartilhado de objetivos. 


\section{Medição de software}

Medição é o processo através do qual números ou símbolos são atribuídos a entidades do mundo real [3] e provê informações quantitativas para o suporte a tomada de decisões, redução dos riscos e aperfeiçoamento contínuo da produção e do produto [5].

Medição é o processo de obtenção de uma medida para uma entidade real.

Uma medida fornece uma indicação de quantidade, dimensão, capacidade ou tamanho de algum produto de software ou de um processo. Em outras palavras uma medida refere-se a um valor de uma métrica. Segundo a norma ISO 9126 [7], métrica é a composição de métodos para medição e escalas de medição.

Para se chegar a uma medida de software existem muitas métricas ${ }^{2}$ que avaliam as variáveis de tamanho, esforço e prazo.

A seguir são descritas as principais características da APF e COCOMO por serem essas métricas utilizadas neste trabalho.

\subsection{Análise por Pontos de Função - APF}

A Análise por Pontos de Função (APF) mede o tamanho do software pela quantificação de suas funcionalidades, baseadas no projeto lógico ou a partir do modelo de dados segundo a visão e os requisitos do usuário final [2].

Suas principais características são: ser independente da tecnologia, ser aplicável desde o início do sistema, apoiar a análise de produtividade e qualidade e estimar o tamanho do software com uma unidade de medida padrão.

Os seguintes passos devem ser observados para mensuração de tamanho do software utilizando esta abordagem [2]: (i) Estabelecer o objeto da contagem (projetos de desenvolvimento, projetos de manutenção ou contagem de uma aplicação); (ii)Determinar a fronteira de medição (a fronteira de medição deve ser sempre determinada sob o ponto de vista do usuário); (iii) Contar as funções de dados, divididos em Arquivos Lógicos Internos (ALIs - que são grupos lógicos de dados mantidos dentro da fronteira da aplicação) e Arquivos de Interface Externa (AIEs - arquivos somente referenciados pela aplicação); (iv) Contar as funções transacionais, divididos em Entradas Externas (EEs), Saídas Externas (SEs) e Consultas Externas (CEs); (v) Determinar o Fator de Ajuste, conjunto de 14 características $^{3}$ que influenciarão a complexidade do software.; e, (vi)Determinar o tamanho do projeto (considera as funções de dados, transacionais, fatores de ajuste e tipo de projeto).

Cada função de dado ou transacional terá um peso diferente dependente de sua complexidade. Diversas tabelas baseadas na quantidade de elementos de dados, de registros e de arquivos referenciados são utilizadas para determinar a complexidade de cada função em Baixa, Média ou Alta.

O resultado da contagem de funções de dados e transacionais é uma medida chamada de contagem não ajustada (NoPF não ajustado), pois não considera detalhes que afetam o produto e sua construção. O ajuste na mensuração é efetuado através do Fator de Ajuste determinado.

\footnotetext{
${ }^{2}$ Ex. LOC, APF, Halstead, COCOMO, COSMIC FFP

${ }^{3}$ Alguma delas: comunicação de dados, processamento distribuído, performance, utilização de equipamento, volume de transações, etc.
} 


\subsection{Abordagem NESMA}

A NESMA (Netherlands Software Metrics Users Association) é uma associação de usuários de métricas que tem proposto alternativas de contagem, utilizando a APF, de forma a possibilitar medir um produto de software no início do processo, mesmo não possuindo todas as informações sobre as funções de dados, transações e características gerais de sistema definidas [10].

A Contagem Indicativa [8] possibilita a estimativa de tamanho a partir de um único componente, os dados (ALI - arquivos lógicos internos e AIE - Arquivos de interface externa), derivando o resto da contagem a partir de bases estatísticas.

Nesta abordagem, considera-se 35 Pontos de Função para cada ALI e 15 Pontos de Função para cada AIE identificado. Os números 35 e 15 representam as médias de pontos de função identificadas para cada um dos tipos de arquivo considerando projetos que, em média, possuam três Entradas Externas(EE), duas Saídas externas (SE) e uma Consulta Externa(CE) para cada ALI e uma Saída Externa (SE) e uma consulta Externa(CE) para cada AIE.

Esta abordagem não prevê a aplicação das 14 Características Gerais de Sistema para a obtenção do fator de ajuste, ou seja, o Fator de ajuste recebe valor de 1.

\subsection{COCOMO}

O COCOMO é um método que busca estimar esforço, prazo, custo e tamanho de equipe, necessários ao desenvolvimento do software, desde que se tenha, como premissa, a dimensão do mesmo. Este método foi desenvolvido por Barry Boehm, a partir de uma pesquisa de campo compreendendo 63 projetos de grande porte, foi proposto em 1981.

Existem 3 modelos neste método [6]:

- COCOMO Básico - versão aplicável a grande maioria de projetos de software: pequenos e médios projetos de software desenvolvidos internamente;

- COCOMO Intermediário - adiciona fatores ao método Básico como restrições de hardware, qualificação, experiência do pessoal e etc;

- COCOMO Detalhado - apresenta técnicas para se estimar tanto a nível de módulo, subsistema e sistema individualizando por fase do projeto.

O COCOMO também categoriza os projetos de software em três tipos fundamentais: Modo Orgânico (Convencional) ${ }^{4}$, Modo Difuso ${ }^{5}$ e Modo Restrito ${ }^{6}$.

Para cada modelo e tipo são definidas equações para estimativas de esforço, prazo e quantitativo de pessoal.

\section{Aplicação da proposta}

\subsection{Breve histórico}

Durante o desenvolvimento dos trabalhos para elaboração do Planejamento Estratégico da instituição, as Áreas de Negócio realizaram um levantamento inicial das suas necessidades

\footnotetext{
${ }^{4}$ Equipes pequenas desenvolvem em um ambiente estável de desenvolvimento, utilizando algoritmos simples e projetos relativamente pequenos.

${ }_{5}^{5}$ Estágio intermediário onde a equipe mescla grande e pouca experiência com a aplicação, com a tecnologia e o tamanho do software médio.

${ }^{6}$ Opera conforme grandes restrições, em um contexto complexo de hardware e software, regras e procedimentos operacionais.
} 
relativas ao desenvolvimento e manutenção de sistemas, visando atender às Diretrizes Estratégicas.

A partir das informações disponibilizadas pelos Gestores a TI teria que:

- Estimar o tamanho, prazo e esforço necessários para o desenvolvimento e manutenção dos sistemas;

- Estimar os custos necessários para o desenvolvimento e manutenção dos sistemas; e,

- Identificar a capacidade produtiva para produzir todas as demandas de sistema solicitadas pelas áreas de negócio.

Considerando estes objetivos foram definidos os seguintes passos: Planejamento, Coleta e Análise de dados.

\subsection{Planejamento}

Para realização dessas estimativas foram estudadas as abordagens APF/NESMA para mensurar o tamanho dos sistemas a serem desenvolvidos e mantidos e o COCOMO para projeção do prazo e equipe necessários.

Para a estimativa do tamanho dos sistemas a serem desenvolvidos para atendimento do Planejamento Estratégico e considerando o grau de imaturidade das demandas a serem cadastradas (impossibilidade de identificação de todos os itens de dados e funções transacionais necessárias para a contagem da APF), foi decidido utilizar a contagem indicativa da NESMA.

Para cálculo das estimativas de prazo e equipe necessária e devido à impossibilidade de levantar dados referentes a restrições de hardware, qualificação, experiência do pessoal, dados com relação à equipe, tecnologia, complexidade, etc., resolveu-se utilizar o COCOMO Básico no Modo Orgânico (convencional).

\subsubsection{Definição de outras estimativas}

A área de tecnologia precisou definir outras estimativas como esforço e custos.

Para estimativa de esforço foi definido utilizar a combinação da APF com a média de produtividade (14,5 horas por Ponto de função) da empresa. A produtividade da empresa é baseada na análise das informações do banco de dados do International Software Benchmarking Standards Group [3]. O foco desse grupo é coletar, validar e publicar um repositório histórico de produtividade em projetos de softwares.

Foi definido utilizar 168 horas como a quantidade de horas produzidas por mês considerando um recurso. Estes valores (produtividade e a quantidade de horas) são utilizados pela empresa em seus contratos de terceirização.

Para a estimativa de custos foi definido utilizar o custo hora de gerentes e técnicos adotado pela instituição e considerar o percentual indicado de esforço (gerencial e técnico) pelas fases da medotologia ${ }^{7}$ adotada pela instituição.

O resumo das estimativas utilizadas esta descrito no Quadro 1.

Quadro 1 - Abordagens definidas para realização das estimativas

\begin{tabular}{|l|l|l|}
\hline Estimativas realizadas & Fórmula base /descrição & Fórmula aplicada \\
\hline Estimativa de tamanho & APF Indicativa (Proposta Nesma) & APF Indicativa \\
\hline $\begin{array}{l}\text { Estimativa de esforço } \\
\text { (homem/mês) }\end{array}$ & $\begin{array}{l}\text { Utilizada média de dados internos para } \\
\text { definição }\end{array}$ & $\begin{array}{l}\mathrm{H} / \mathrm{M}=\left(\mathrm{PF} \times 14,5^{*}\right) / 168 \\
{ }^{*} \text { Qtd horas por ponto de função }\end{array}$ \\
\hline Estimativa de prazo em & COCOMO Básico/Modo Orgânico & A abordagem COCOMO como indicada \\
\hline
\end{tabular}

\footnotetext{
${ }^{7}$ A metodologia da empresa identifica por fase um percentual de esforço técnico e gerencial.
} 


\begin{tabular}{|c|c|c|}
\hline Estimativas realizadas & Fórmula base /descrição & Fórmula aplicada \\
\hline meses & Prazo em meses $=2.5 \times(\mathrm{H} / \mathrm{M})^{\wedge} 0,38$ & Prazo em meses $=2.5 \times(\mathrm{H} / \mathrm{M})^{\wedge} 0,38$ \\
\hline $\begin{array}{ll}\text { Estimativa } & \text { de número } \\
\text { médio de } & \text { profissionais } \\
\text { necessários } & \end{array}$ & $\begin{array}{l}\text { COCOMO Básico/Modo Orgânico } \\
\text { Equipe }=\quad \text { Estimativa de } \\
\text { esforço/Estimativa de prazo em meses }\end{array}$ & $\begin{array}{l}\text { A abordagem COCOMO como indicada } \\
\text { Equipe = Estimativa de esforço/Estimativa } \\
\text { de prazo em meses }\end{array}$ \\
\hline Estimativa de custos & $\begin{array}{l}\text { Utilizadas média de custos internos } \\
\text { para definição com relação à hora } \\
\text { trabalhada de técnicos/gerentes e etc } \\
\text { Utilizados percentuais de esforço } \\
\text { dentro da metodologia de técnicos e } \\
\text { gerentes }\end{array}$ & $\begin{array}{l}\text { Custos }=\text { (custo hora gerencial } \mathrm{x} \% \text { esforço } \\
\text { gerencial nas fases da metodologia) }+ \\
\text { custo hora técnico } \mathrm{x} \% \text { esforço técnico nas } \\
\text { fases da metodologia) }\end{array}$ \\
\hline
\end{tabular}

\subsection{Coleta de Dados}

\subsubsection{Definição do aplicativo a ser desenvolvido}

Para que os gestores das áreas de negócio pudessem cadastrar as suas necessidades, foi desenvolvida uma aplicação para o detalhamento dos Planos de Tecnologia que atenderiam as Ações Operacionais ${ }^{8}$ de sua área identificando as demandas de desenvolvimento ou manutenção em sistemas já existentes. Foram solicitadas informações sobre: qtd arquivos lógicos (tabelas), qtd arquivos de interface externa (interfaces), projeto novo ou já demandado a tecnologia, se já demandado número identificador da solicitação, qtd consultas, etc.

É interessante observar que alguns dados coletados não foram utilizados para o cálculo, mas serviram na identificação do grau de amadurecimento do gestor com relação à demanda.

Os gestores das áreas de negócio foram treinados na utilização da ferramenta e a mesma foi disponibilizada na Intranet da empresa durante um período determinado de tempo.

A responsabilidade pela qualidade dos dados informados ficou a cargo dos gestores das áreas de negócio.

\subsection{Análise dos dados}

\subsubsection{Demandas Cadastradas}

Foram cadastradas 931 demandas, destas, 16 tinham término previsto ainda para o ano de 2003 e foram desconsideradas, pois o foco do trabalho era o planejamento de 2004. Para efeito deste estudo, foram consideradas as 915 demandas restantes.

Essas demandas foram classificadas conforme Quadro 2 :

Quadro 2 - Classificação de demandas

\begin{tabular}{|l|l|}
\hline Novo desenvolvimento $^{9}$ & Novas demandas $^{10}$ \\
\cline { 2 - 2 } & Demandas em andamento $^{11}$ \\
\hline Desenvolvimento evolutivo $^{12}$ & Novas demandas \\
\cline { 2 - 2 } & Demandas em andamento \\
\hline
\end{tabular}

Conforme pode ser verificado na Figura 1, do total de demandas cadastradas, 677 demandas são novas, correspondentes a $75 \%$ do total, e $25 \%$ já estavam em andamento.

\footnotetext{
${ }^{8}$ Ações operacionais - Subgrupo de ações vinculadas às diretrizes estratégicas

${ }^{9}$ Identificação de necessidade de desenvolvimento de novos sistemas

${ }^{10}$ Demandas de software ainda não solicitadas a TI

${ }^{11}$ Demandas de software já solicitadas a TI

${ }^{12}$ Identificação da necessidade de uma manutenção evolutiva em um sistema já existente
} 


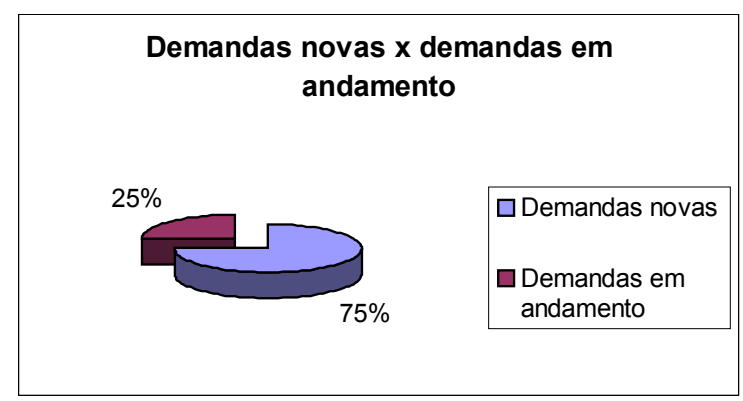

Figura 1 - Demandas novas $x$ demandas em andamento

A Tabela 1 e Figura 2 demonstram a quantidade de demandas cadastradas com relação à sua classificação, novo desenvolvimento ou desenvolvimento evolutivo.

Tabela 1 - Classificação das demandas

\begin{tabular}{|l|l|l|l|l|l|l|}
\hline \multirow{2}{*}{ Demanda } & \multicolumn{2}{|l|}{ Demandas em andamento } & Novas Demandas & \multicolumn{3}{l|}{ Não identificadas } \\
\cline { 2 - 8 } & QTD & $\%$ & QTD & $\%$ & QTD & $\%$ \\
\hline Novo desenvolvimento & 75 & $24 \%$ & 228 & $73 \%$ & 6 & $1,9 \%$ \\
\hline Desenvolvimento evolutivo & 151 & $24 \%$ & 449 & $74 \%$ & 6 & $1 \%$ \\
\hline Total & 226 & & 677 & & 12 & \\
\hline
\end{tabular}

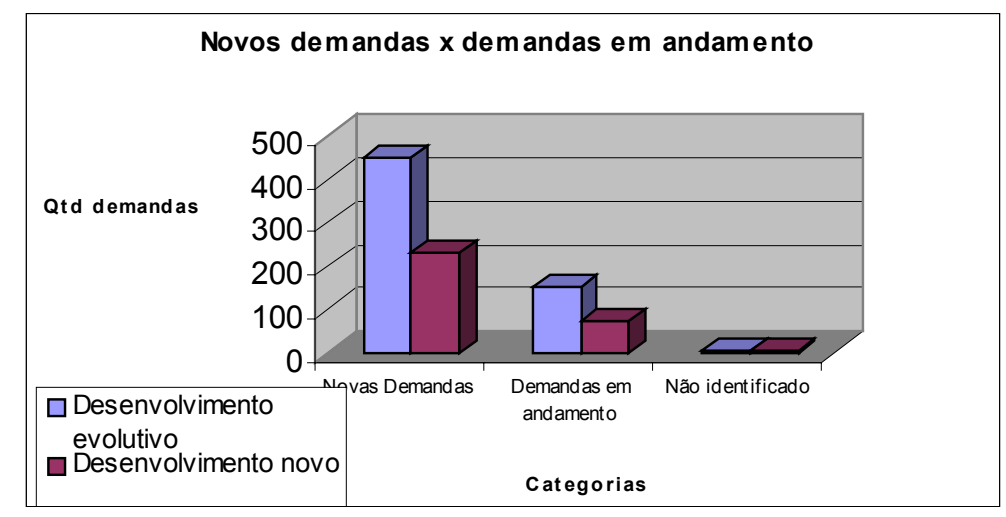

Figura 2 - Novas demandas $\mathrm{x}$ demandas em andamento

Estas análises foram importantes, pois identificaram que a maior parte das demandas de produtos de TI (75\%) para atender ao Planejamento Estratégico seriam novas demandas e a TI teria que se preparar para conseguir produzir bem mais do que vinha produzindo.

\subsubsection{Tamanho e custo das demandas cadastradas}

Para analisar o tamanho das demandas cadastradas foi utilizada a classificação já existente na Metodologia de Desenvolvimento de Sistemas da empresa que prevê três classes de projeto (até $105 \mathrm{PF}$, até $525 \mathrm{PF}$ e acima de $525 \mathrm{PF}$ ). Essas classificações foram obtidas no mercado e identificam respectivamente projetos pequenos, médios e grandes. Os quantitativos são apresentados na Figura 3. 


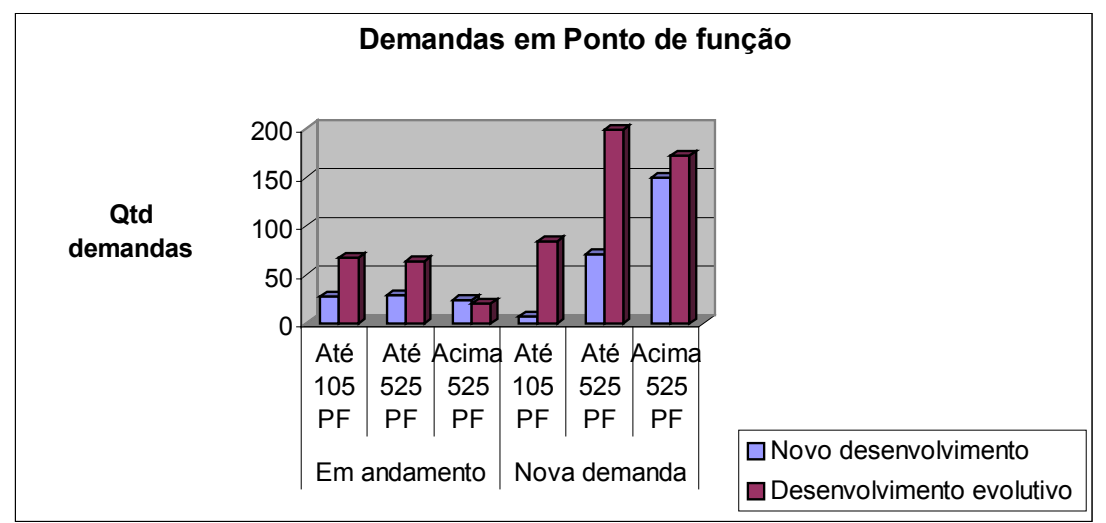

Figura 3 - Classificação das demandas

Após a análise dos dados coletados foi identificado que, das demandas inseridas como novas (não em andamento), 35\% (322 demandas) estavam acima de 525 PF (valor considerado pela TI, para sistemas de desenvolvimento superiores a 6 meses). Foi decidido, então, aplicar o COCOMO como apresentado anteriormente, para identificar: o prazo total em meses, o número de profissionais necessários e o custo estimado para o ano de 2004 e para os demais anos.

Como resultado, concluiu-se que se todas as demandas fossem iniciadas em 2004, $68 \%$ poderiam ser terminadas em 2004 utilizando-se um total de 3.169 recursos, e a um custo projetado conforme demonstrado na tabela. O restante das demandas seria concluído no ano de 2005/2006 devido à estimativa de prazo ultrapassar um ano, conforme demonstrado na Tabela 2.

Tabela 2 - Estimativa 2004/2005/2006

\begin{tabular}{|l|l|l|l|l|l|}
\hline & 2004 & 2005/2006 \\
\hline & $\begin{array}{l}\text { Qtd. } \\
\text { Recursos } \\
\text { necessários }\end{array}$ & Valor projetado & $\begin{array}{l}\text { Qtd PF } \\
\text { projetada }\end{array}$ & Valor projetado & $\begin{array}{l}\text { Qtd PF } \\
\text { projetada }\end{array}$ \\
\hline Novas demandas & 3.169 & $271.264 .472,00$ & 346.205 & $120.059 .225,00$ & 156.205 \\
\hline Demandas em andamento & & $61.063 .600,00$ & 78.523 & $19.796 .997,00$ & 25.950 \\
\hline
\end{tabular}

Para as demandas em andamento, não foi necessário estimar o número de profissionais uma vez que são demandas que já estão sendo atendidas pela TI.

\section{Conclusões}

A TI precisa constantemente estimar a necessidade futura de produção de sistemas da empresa para conseguir alinhar seus objetivos com os objetivos estratégicos da organização.

Neste trabalho foram utilizadas métricas (APF e COCOMO) para identificar a capacidade produtiva necessária para a TI estar alinhada ao Planejamento Estratégico de uma instituição financeira do governo federal.

Nem todas as informações foram identificadas através da aplicação destas métricas, mas os resultados obtidos representam, no momento, um conjunto de indicadores que foram e poderão ser utilizados para análise da situação atual do processo. Segundo Fenton e Pfleeger [4] mensura-se para entender, controlar e aperfeiçoar o processo de produção de software e conseqüentemente adicionar qualidade.

Um grande benefício identificado pela área de tecnologia foi o de poder saber, com a tempestividade requerida, as demandas que serão solicitadas nos próximos dois anos e poder trabalhar na definição de estratégias para o seu atendimento, incluindo: definições sobre 
níveis de terceirização, contratação de mão-de-obra, treinamento e capacitação de equipes, aquisição de novas tecnologias e etc.

Com base nesses dados, a área de TI pôde se posicionar junto à organização informando sua capacidade contratual atual (terceirização), gerencial e de recursos técnicos e elaborar comparativos entre a capacidade produtiva atual e a estimada. Nestes comparativos verificou-se a impossibilidade da TI atender a todas as demandas identificadas. Com base nesses resultados, a empresa decidiu priorizar, em um segundo momento, as ações operacionais, pois seria praticamente inviável o atendimento a todas as demandas cadastradas nos planos de tecnologia.

É interessante ressaltar que as contagens efetuadas, com as abordagens adotadas, são estimativas iniciais e que a TI deve acompanhar e atualizar estas contagens até a entrega dos produtos de forma a manter um histórico da evolução e melhorar continuamente suas estimativas iniciais.

É aconselhável que esse processo seja melhorado e recomenda-se uma maior participação dos gestores de negócio e das equipes de desenvolvimento de software para que, num esforço conjunto, a idéia de identificar as demandas de software requeridas para atendimento ao Planejamento Estratégico passe a ser vista como um benefício para a empresa e seja realizada com maior consciência e qualidade.

\subsection{Trabalhos futuros}

Estudos de outras abordagens que poderiam ser utilizadas visando o alinhamento estratégico de TI com o alinhamento estratégico da empresa.

Análise futura e comparação das pontuações dos sistemas identificados nessa primeira coleta que forem efetivamente desenvolvidos, visando uma calibração dos percentuais.

\section{Bibliografia}

[1] BOAR, Bernard: Tecnologia da Informação: A arte do planejamento estratégico. São Paulo: Berkeley 2002. 329 p.

[2] IFPUG. International Function Point Users Group. Function Point Counting Practices Manual: Release 4.1. Ohio: IFPUG. 2000. 1 v.

[3] ISBSG. Benchmarking Repository, Release 6. ISBSG. Abr, 2002.

[4] FEnTON,N., PFLEEGER, S. Software Metrics A Rigorous \& Practical Approach. Boston: PWS Publishing Company, 1997. 638 p.

[5] FENTON, N., NEIL, M. Software Metrics: Roadmap. Future of Software Engineering Limerick Ireland ACM. p. 359-370, 2000.

[6] FERNANDES, A . Gerência efetiva de software através de métricas.São Paulo: Atlas, 1995.

[7] ISO/IEC 9126-1. Software engineering - Product quality. 2001.

[8] NESMA. Early Function Point Counting. Disponível em: www.nesma.nl/enlish/earlyfpa.htm. Acesso em 16/9/03

[9] TAURION, César. Visão de mercado: estratégia e negócios na Internet - tecnologia não faz milagre. Maio, 2002. Disponível em: <http://www.timaster.com.br/revista/ colunistas/ler_colunas_emp.asp?cod=562.> Acesso em 16/9/02.

[10] VAZQUEZ, C., SIMÕES, G., ALBERT, R. Análise de Pontos de Função, Medição, Estimativas e Gerenciamento de Projetos de Software. 1a. ed, São Paulo, Erica, 2003. 